\title{
Criminologie
}

\section{La Sûreté du Québec des origines à nos jours : quelques repères historiques}

\section{Jean-François Leclerc}

Volume 22, numéro 2, 1989

Le milieu criminel

URI : https://id.erudit.org/iderudit/017284ar

DOI : https://doi.org/10.7202/017284ar

Aller au sommaire du numéro

\section{Éditeur(s)}

Les Presses de l'Université de Montréal

ISSN

0316-0041 (imprimé)

1492-1367 (numérique)

Découvrir la revue

Citer cet article

Leclerc, J.-F. (1989). La Sûreté du Québec des origines à nos jours : quelques repères historiques. Criminologie, 22(2), 107-127.

https://doi.org/10.7202/017284ar
Résumé de l'article

The history of the police in Canada is a field that has been little explored. This is all the more so in the case of the police corps of Quebec, the only valid monograph on the subject being one on the Montreal police. This article gives a general outline of the administrative history of the provincial police of Quebec the "Sûreté du Québec", since its creation in 1870 by the provincial government. The idea of creating a police force under State control was not new, going back to the first «modern» police established in 1838 in Quebec city and Montreal during a time of rebellion, by an Order in Council of governor Durham. An unsuccessful attempt was made to establish one during the 1850s. It was in 1870 that the "provincial police» were set up in Quebec City, the capital, mainly to have a force to intervene in riots and strikes. Municipalities requiring them could also obtain their services. This police corps was reduced after 1878 to become a mere guard of the Parliament which also served the Department of the Attorney General. After that, great changes were made with the integration of other police and government services : the Bureau of Provincial Detectives of Montreal in 1922, the liquor and highway police in 1934 and 1936, which in 1938 become branches of the Provincial Police. In 1938, the Duplessis government undertook to extend the provincial police by opening up stations throughout the province. After 1960, the Lesage government completed the merging of the various branches, sought to eliminate political influence in the hiring of police and opened the first training school for policemen. The Provincial Police became the Sûreté du Québec in 1968, when an administrative restructuring was started, inspired by the methods used in private enterprise, which gave its form to today's Sûreté.
Tous droits réservés @ Les Presses de l'Université de Montréal, 1989
Ce document est protégé par la loi sur le droit d'auteur. L’utilisation des services d’Érudit (y compris la reproduction) est assujettie à sa politique d'utilisation que vous pouvez consulter en ligne.

https://apropos.erudit.org/fr/usagers/politique-dutilisation/ 
LA SÛRETÉ DU QUÉBEC DES ORIGINES À NOS JOURS : QUELQUES REPÈRES HISTORIQUES Jean-François Leclerc*

The history of the police in Canada is a field that has been little explored. This is all the more so in the case of the police corps of Quebec, the only valid monograph on the subject being one on the Montreal police. This article gives a general outline of the administrative history of the provincial police of Quebec, the "Sûreté du Québec", since its creation in 1870 by the provincial government. The idea of creating a police force under State control was not new, going back to the first «modern" police established in 1838 in Quebec city and Montreal during a time of rebellion, by an Order in Council of governor Durham. An unsuccessful attempt was made to establish one during the 1850s. It was in 1870 that the «provincial police» were set up in Quebec City, the capital, mainly to have a force to intervene in riots and strikes. Municipalities requiring them could also obtain their services. This police corps was reduced after 1878 to become a mere guard of the Parliament which also served the Department of the Attorney General. After that, great changes were made with the integration of other police and government services: the Bureau of Provincial Detectives of Montreal in 1922, the liquor and highway police in 1934 and 1936, which in 1938 become branches of the Provincial Police. In 1938, the Duplessis government undertook to extend the provincial police by opening up stations throughout the province. After 1960, the Lesage government completed the merging of the various branches, sought to eliminate political influence in the hiring of police and opened the first training school for policemen. The Provincial Police became the Sûreté du Québec in 1968, when an administrative restructuring was started, inspired by the methods used in private enterprise, which gave its form to today's Sûreté.

\section{L'HISTOIRE DE LA POLICE : UN CHAMP PEU EXPLORÉ}

L'institution policière reçoit depuis longtemps une attention particulière de la part des sciences sociales. L'abondante littérature savante sur ce sujet en témoigne de façon éloquente. Pourtant, parmi les chercheurs qui se sont intéressés à la police, bien peu ont porté sur elle un regard rétrospectif. Cela est particulièrement flagrant dans le cas du Canada, où l'histoire des corps de police demeure un champ de

\footnotetext{
* L'auteur est historien.
} 
recherche peu exploré, sinon par l'institution policière elle-même, et la plupart du temps pour des fins commémoratives ${ }^{1}$.

Comme le montre une bibliographie récente ${ }^{2}$, la majeure partie de la production historique sur la police au Canada depuis un siècle est en effet constituée de récits anecdotiques, de recueils de souvenirs, et de quelques historiques de service ${ }^{3}$, très rarement d'études réalisées par des chercheurs des sciences sociales ${ }^{4}$. De plus, la moitié environ des ouvrages et articles recensés sont consacrés à la Gendarmerie royale du Canada, et les monographies sur les corps de police provinciaux et municipaux sont rares. La production historique québécoise sur la police se résume à peu de choses : à l'exception de quelques pages ou paragraphes dans les ouvrages portant sur des sujets connexes ${ }^{5}$, l'unique ouvrage valable sur l'histoire d'un corps de police québécois demeure encore aujourd'hui le travail déjà ancien de Jean Turmel sur la police de Montréal, en deux tomes qui couvrent les années 1796 à 19716. Pas

1. Du côté américain, le questionnement historique sur la police est beaucoup plus ancien. Citons par exemple les ouvrages de Raymond B. Fosdick, American Police Systems, (Montclair, New Jersey, Patterson Smith, 1920, 1969), et de Bruce Smith, The State Police Organization and Administration, (Montclair, New Jersey, Patterson Smith, 1925,1969 ). Il fut associé au mouvement de réforme des administrations municipales et de la police que connaissait ce pays depuis le début du siècle. À ce sujet, on peut consulter Robert M. Fogelson, Big-City Police, Harvard University Press, Cambridge, Massachusetts and London, England, 1977.

2. Russell C. Smandych, Catherine J. Mathews, Sandra J. Cox, Canadian Criminal Justice History. A Annotated Bibliography, University of Toronto Press, Toronto Buffalo London, 1987.

3. Ce genre demeure encore de nos jours le plus populaire, comme le montre la publication très récente d'une volumineuse histoire de l'Ontario Provincial Police, écrite par un officier de ce corps de police. O.P.P. The History of the Ontario Provincial Police Force, by Dahn D. Higley, The Queen's Printer, Toronto, 1984.

4. Mentionnons celles de G. Marquis, «A Machine of Oppression Under the Guise of the Law»: The Saint John Police Establishment, 1860-1890», Acadiensis; de N. Rogers, «Serving Toronto the Good : The Development of the City Police Force, 18341884», dans Victor Russell éd., Forging a Consensus : Historical Essays on Toronto, Thronto, 1984.

5. Par exemple sur la police municipale de Québec dans G.-H. Dagneau, G.-H. et al, La ville de Québec, histoire municipale, $N$, de la Confédération à la charte de 1929. La Société historique de Québec, 1983; Drolet, A., La Ville de Québec, histoire municipale III - de l'incorporation jusqu'à la Confédération (1833-1867). La Société historique de Québec, Québec, 1967; aussi, J.-M. Fecteau, La pauvreté, le crime, l'Etat, essai sur l'économie politique du contrôle social au Québec 1791-1840, Thèse de doctorat, Paris VII, 1983.

6. J. Turmel, Historique de Service 1796-1909. Premières structures et évolution de la police de Montréal. Document interne, Service de la Police de Montréal, 1971; Police de Montréal. Historique du service 1909-1971, document inteme, Service de la Police de Montréal, 1974. Son analyse a cependant le défaut de se pencher un peu lourdement sur l'évolution des structures au détriment du contexte pourtant riche de l'histoire politique montréalaise. 
étonnant dans ces conditions que la police québécoise trouve peu de place dans une récente synthèse historique sur le système de police canadien?

Le présent article se situe dans ce vacuum. Il se propose de donner quelques points de repère sur l'histoire de la Sûreté du Québec, alors que s'achève une recherche entreprise il y a trois ans à la demande de cette institution ${ }^{8}$. L'objectif de cette recherche était de produire une histoire administrative et professionnelle de la Sûreté, c'est-à-dire de décrire et dater, en les fondant sur des sources d'archives, les principales transformations internes de la Surreté du Québec. Ces limites ne permettaient pas d'aborder en profondeur les rapports complexes et changeants de l'institution policière avec la société, le droit et le pouvoir politique. Le seul travail pour retrouver les documents administratifs de la Sûreté fut d'ailleurs considérable. En effet, la Sûreté n'a pas conservé dans ses murs des archives administratives antérieures aux années 1970-80. Il fallut donc retracer les dossiers la concernant parmi la volumineuse correspondance du ministère de la Justice à Québec, c'est-à-dire des milliers de dossiers administratifs et criminels déposés aux Archives nationales à Québec et au Centre de pré-archivage du ministère. Cette tâche était lourde mais indispensable: à la différence des autres services de l'administration publique, que James lain Gow a pu étudier exclusivement à partir de publications officielles, documents publics gouvernementaux et monographies ${ }^{9}$, la police de la province fut presque absente des sources officielles jusqu'à récemment, sauf par les rapports annuels publiés à la fin du $19^{\mathrm{e}}$ siècle et à nouveau à partir des années 1960, les comptes publics de la province, les lois la concernant, et une commission royale d'enquête ${ }^{10}$. Se contenter de ces seuls documents nous exposait à des redites et à des imprécisions ${ }^{11}$. Le dépouillement des documents internes, des dossiers du personnel, de la

7. C.K. Talbot, C.H.S. Jayewardene, et T.J. Juliani, Canada's Constables, The Historical Development of Policing in Canada, Crimcare, Ottawa, 1985.

8. Une «Histoire de la Sûreté du Québec» devrait être publiée dans les mois qui suivent. Nos remerciements à J. Raymond Proulx pour sa collaboration à la recherche.

9. J.I. Gow, Histoire de l'administration publique québécoise 1867.1970, Les Presses de l'Université de Montréal, l'Institut de l'administration publique du Canada, 1986. Voir l'introduction, chapitre 1, p.14.

10. Commission royale d'enquête sur les activités dans le district de Montréal, de la Police provinciale, et de la Police des liqueurs, (1944).

11. Un seul des rapports de recherche cités dans la bibliographie de Gow (op.cit.) porte sur la police provinciale. (Boris Domazet et Jean Castonguay, «Histoire administrative de la Police provinciale du Québec, 1870-1945», Département des sciences politiques, Université de Montréal 1972, 32 pages et annexe). Parce qu'ils puisaient exclusivement dans les sources officielles et publiées, les auteurs avouent avoir été contraints à faire une «analyse de kangourou». 
correspondance, des journaux et d'ouvrages sur l'histoire du Québec s'imposait. Il nous permit d'obtenir un portrait plus exact, documenté, de l'évolution de la Sûreté du Québec dont les pages qui suivent traceront les grandes lignes 12 .

\section{0-1878 : UNE GENDARMERIE PROVINCIALE LONGTEMPS ATTENDUE13}

Le 23 décembre 1869, l'Assemblée législative de la province de Québec, à majorité conservatrice, adopte une loi intitulée «Acte pour établir un système général de police en cette province» 14 . Elle a pour effet de créer un corps de police relevant directement du gouvernement provincial ${ }^{15}$ et destiné à intervenir lors d'émeutes électorales, religieuses et de conflits ouvriers tout en veillant au respect des lois fédérales et provinciales, et des règlements municipaux ${ }^{16}$. La sanction de la loi le $1^{\text {er }}$ février 1870 est suivie par la nomination d'un commissaire de police, Pierre-Antoine Doucet, juge des sessions de Québec, et de deux surintendants ${ }^{17}$, ainsi que d'une soixantaine d'hommes qui commencent en mai suivant à patrouiller les rues de la ville de Québec, où ils font office de police municipale. En effet, la loi ne cherche pas seulement à former un corps de police d'élite dans la capitale; elle veut établir un "système général de police» où toute municipalité désireuse d'établir une police sur son territoire pourrait retenir les services des policiers provinciaux, temporairement ou en permanence. Leur entretien serait alors défrayé partiellement par l'État. Si la province subventionne la police des municipalités qui adhèrent au système, celles-ci perdent en retour leur droit de gérance sur la police. En effet, les policiers détachés pour faire respecter la réglementation municipale, en

12. L'ouvrage en préparation abordera des questions qui ne sont pas touchées ici, ou très peu, comme l'influence politique dans l'embauche, les interventions lors de grèves. Notez qu'en raison de la plus grande dispersion des archives pour les décennies 1970-1980 et de l'abondance de la documentation, des publications savantes, compte tenu du temps à notre disposition, la période contemporaine sera esquissée à grands traits.

13. Cette section a fait l'objet en mars 1987 d'une rencontre du Groupe d'étude sur I'histoire du contrôle social, à l'Université de Montréal, sous le titre "Genèse et naissance d'une police d'État — La Police provinciale de Québec (1870)». chap. 24.

14. Acte pour établir un système général de police en cette province, 33 Vict.

15. Par son secrétaire et registraire.

16. Voir les débats à ce sujet. Assemblée nationale du Québec, débats de l'Assemblée législative, lère législature, $3^{e}$ session, texte établi par Marcel Hamelin, Québec, Journal des Débats, 1974. (Ci-après DALQ).

17. Ludger-Napoléon Voyer, ancien militaire et capitaine dans les Voltigeurs de Québec; Henry Heigham, officier de l'armée britannique. 
même temps que les lois provinciales, sont nommés et dirigés par le quartier général de Québec. On espère de la sorte placer la police sous un patronage plus éclairé que celui des municipalités, identifié par plusieurs comme une cause de la piètre qualité des polices urbaines ${ }^{18}$. L'adhésion au système de police provincial n'est cependant pas obligatoire, sauf dans la capitale où la police municipale est déjà partiellement sous contrôle gouvernemental ${ }^{19}$. Québec, centre du système de police, doit assurer à la police provinciale un financement et un effectif minimaux. Malgré les critiques de l'opposition libérale à l'Assemblée législative et des représentants de la ville de Québec concernant l'inutilité de la loi, son inconstitutionnalité et son caractère centralisateur, le projet de loi qui est adopté le 23 décembre 1869 est sanctionné le $1^{\text {er }}$ février 1870 .

Le «système de police» établi en 1870 met en oeuvre un projet entretenu depuis plusieurs décennies par les autorités du Canada-Uni, à une époque où se formaient les institutions de l'État canadien. En effet, l'idée de mettre sur pied une police qui aurait le monopole des tâches policières sur le territoire n'est pas nouvelle. Les émeutes électorales, ethniques et religieuses, et les conflits ouvriers qui éclataient périodiquement à Québec et à Montréal n'avaient cessé de démontrer les faiblesses, en temps de crise, du système de contrôle essentiellement réactif hérité de l'Angleterre après la Conquête ${ }^{20}$. En effet, depuis les années 1820-1830, comme nombre de cités américaines et anglaises de cette époque, Montréal et Québec éprouvaient des problèmes liés à une forte immigration européenne et aux transformations de l'économie21.

18. En 1862, dans son rapport annuel, le chef de police de la ville de Québec «suppliait», c'est son expression, le conseil municipal de lui donner le pouvoir de choisir et de renvoyer les policiers, déplorant les pressions exercées par des citoyens «de mérite et du caractère religieux et moral le plus élevé» en faveur "de personnes aux moeurs tellement dépravées qu'elles étaient tout à fait incapables de veiller à la conservation de la paix publique». Rapport annuel du chef de police, Comptes du trésorier de la Cité et autres documents de la Corporation de Québec pour l'année 1862.

19. La gestion de la police municipale est confiée depuis 1865 à un «Bureau de police" composé du juge provincial (le juge des Sessions), du juge municipal (le recorder), et du maire de la ville. Plusieurs corps de police du Canada et des Etats-Unis, entre autres ceux de New-York et de Toronto, vivaient sous le même régime.

20. Ce système était fondé sur le pouvoir dissuasif et répressif des tribunaux, et sur l'action des juges de paix bénévoles, de quelques officiers de justice (les constables et grands constables), des petits corps de garde urbains, les hommes de guet (une vingtaine à Québec et à Montréal) et sur la force brute de l'armée. À ce sujet, on peut consulter Jean-Marie Fecteau, op.cit.

21. Sur les polices anglaises et américaines, voir CRITCHLEY, T.A., History of Police in England and Wales, Patterson Smith, New Jersey, 1972 ; S.L. Harring. Policing a Class Society: The Experience of American Cities, 1865-1915, New Brunswick Rutgers University Press, 1983 ; Wilbur R. Miller, Cops and Bobbies : Police authority in New York and London 1830-1870, University of Chicago Press, Chicago, 1977. 
Le système aurait probablement évolué lentement si l'effervescence patriote et les insurrections de 1837-38 n'avaient complètement paralysé les instances locales de contrôle. Au cours du bref séjour du Lord enquêteur Durham, en 1838, et par la suite sous la tutelle du commandant militaire Colborne, les autorités de la colonie mettaient sur pied dans les villes de Québec et de Montréal, en l'absence de tout assentiment démocratique ${ }^{22}$, des polices préventives et salariées: une police urbaine d'une centaine d'hommes dans chaque ville, une police rurale d'égale importance pour la région de Montréal. Placées sous l'autorité du Secrétaire civil gouvernemental et de magistrats stipendiaires, ces polices devaient maintenir l'ordre dans les rues, prévenir le crime mais aussi surveiller les activités politiques des populations rebelles et de leurs élites. C'est donc à leur révolte que les habitants du Bas-Canada devaient de voir une première police «modeme» patrouiller les rues et chemins bien avant la plupart des villes de l'Angleterre (hormis Londres) et des États-Unis ${ }^{23}$. Les modèles métropolitains qui avaient inspiré ces innovations étaient et devaient rester encore longtemps le nec plus ultra en matière de police: la Metropolitan Police de Londres, une police civile mise sur pied en 1829 et placée sous le contrôle du Home Secretary, et le Irish Constabulary Force, établie progressivement dès la fin du $18 \mathrm{e}$ siècle, et plusieurs fois restructurés pour mieux pacifier les populations rebelles d'Irlande 24 .

Après l'écrasement des rébellions, et l'Union des deux Canada en 1840 , les villes de Montréal et de Québec avaient retrouvé, avec leurs chartes, le contrôle de leur police ${ }^{25}$. Une fois dissoute la police gouvernementale urbaine et rurale, l'État avait repris une place plus discrète

22. L'Assemblée législative du Bas-Canada avait été dissoute en 1836 et remplacée par un conseil spécial non élu. Les corporations municipales sont aussi abolies, leurs chartes n'ayant pas été renouvelées.

23. En Angleterre, le gouvernement fut longtemps indifférent à la police hors de Londres. Les villes incoporées sont habilitées à former des polices en 1835, sans y être obligées; les comtés jouissent de ce pouvuir à compter de $i 839$, mais ne seront contraints à en user qu'en 1856. Aux États-Unis, New-York sera en 1845 la première ville à former un corps de police urbain. Voir T.A. Critchley, History of Police in England and Wales, Patterson Smith, New Jersey, 1972; Wilbur R. Miller, Cops and Bobbies: Police Authority in New York and London 1830-1870, University of Chicago Press, Chicago et Londres, 1977.

24. Ajoutons que selon E.K.Senior, un autre modèle serait celui de la police instaurée en Jamaïque a la suite d'une révolte d'esclaves. "The influence of the British Garrison on the Development of the Montreal Police, 1832 to 1853 ", Military Affairs, April 1979, p.63-68. Au sujet de la police irlandaise, voir S.H. Palmer, Police and Protest in England and Lreland 1780-1850, Cambridge University Press, 1988.

25. Les chartes d'incorporation leur sont octroyées en 1840 , et le contrôle de la police à la fin de 1842. Des corps de police municipaux sont mis sur pied au printemps de 1843. 
en matière de police. Il mettait sur pied des polices spéciales et temporaires pour faire face à des situations d'urgence, par exemple en 1849 lors de l'incendie du Parlement à Montréal par des émeutiers, ou pour surveiller des activités se déroulant hors des territoires municipaux, comme les chantiers de construction des canaux de Lachine et Beauharnois au cours des années 1840 , l'exploitation minière dans la Beauce, la navigation fluviale et les activités portuaires ${ }^{26}$.

La recherche d'une solution globale aux questions de sécurité n'était pas abandonnée pour autant, à une époque où les émeutes urbaines démontraient l'inefficacité des corps de police municipaux à contrôler les foules. En 1853 et 1854 , réagissant à des émeutes religieuses à Montréal et Québec, alors la capitale, deux commissions d'enquête du gouvernement avaient élaboré le projet ambitieux d'une police d'État centralisée, casernée, version hybride des modèles irlandais et londonien. On la concevait comme le rouage intérieur d'un système de défense fondé en outre sur une milice et une armée ${ }^{27}$. Ces recommandations avaient été reprises dans un projet de loi de 1856 intitulé «Bill pour établir un système général de police en cette Province, et une force de police suffisante dans certaines cités et villes et autres places où les autorités municipales le requerront». Mais devant les protestations de Montréal, Québec, Toronto et de plusieurs autres municipalités, à une époque d'instabilité ministérielle, le projet avait été retiré au moment de la seconde lecture. Les solutions de rechange furent de mieux organiser la milice, d'augmenter le nombre de districts judiciaires (donc, des officiers de justice exerçant certaines fonctions policières, comme le Grand constable), et enfin, à l'exemple de plusieurs états américains, de confier l'embauche et la discipline de certains corps de police à des Bureaux de police indépendants formés de représentants gouvernementaux et municipaux ${ }^{28}$.

À la fin des années 1860 , la conjoncture s'était avérée plus favorable, semble-t-il, au projet d'une police canadienne centralisée. En effet, les inquiétudes causées par la guerre civile américaine, et l'acqui-

26. Ces polices étaient mises sur pied pour certaines fins particulières en vertu des lois réglant les travaux publics, l'exploitation minière, le commerce portuaire et la navigation fluviale. Voir C.K. Talbot, C.H.S. Jayewardene, et T.J. Juliani, op.cit.

27. Rapport des commissaires nommés pour faire une enquête sur la conduite des autorités de police lors de l'émeute de l'église Chalmers, le 6 juin 1853, avec les minutes des procédés et des témoignages rendus devant ladite commission. Imprimerie de Louis Perrault, Québec, 1854 ; Rapport des commissaires nommés pour s'enquérir et faire rapport des meilleurs moyens de réorganiser la milice en Canada, et pour rapporter un plan perfectionné de police, Stewart Derbishire \& Georges Desbarats, Québec, 1855.

28. En 1856, la ville de Toronto proposa cette solution qu'elle préférait à une tutelle gouvemementale complète. N. Rogers, op.cit. 
sition des vastes territoires de l'Ouest demandaient de reconsidérer la question $^{29}$. De plus, le retrait de l'armée britannique du pays était imminent, ce qui allait priver le pays d'un recours essentiel en temps de crise ${ }^{30}$. Voilà pourquoi à l'aube de la Confédération, entre 1868 et 1871,1 'idée de former des polices gouvernementales refait surface dans les officines du gouvernement fédéral et des gouvernements provinciaux, ceux-ci ayant en vertu de la Constitution la responsabilité d'appliquer les lois et de maintenir l'ordre sur leur territoire. En 1868, une loi fédérale crée la "police du Dominion» qui doit veiller à la garde des édifices parlementaires et fédéraux, et au respect de certaines lois nationales ${ }^{31}$; au même moment, à l'instigation du premier ministre John A. Macdonald, on entreprend d'établir une police à cheval pour le far-west canadien, la North West Mounted Police ${ }^{32}$. Dans les provinces d'Ontario et du Québec, c'est en 1869 que sont présentés des projets de lois pour créer des polices provinciales, alors que les autres provinces font de même en 1870 et $1871^{33}$. La législature ontarienne ayant cédé aux pressions des autorités municipales ${ }^{34}$, le projet québécois d'une police d'État est le premier à se concrétiser au Canada, quatorze ans après le projet de loi de Macdonald dont il s'inspirait ${ }^{35}$.

Le «système général de police» établi par le Québec en 1870 n'obtient pas auprès des municipalités l'effet escompté par le législateur. Si la police provinciale se mérite des éloges, même dans la presse montréalaise qui loue sa tenue et son efficacité lors des émeutes et des

29. Mentionnons entre autres les projets belliqueux des «Fenians», des activistes américains d'origine irlandaise, contre le Canada britannique. En 1866 et en 1870, ils font des incursions armées qui sont repoussées par la milice.

30. PARISEAU, J., L'Aide militaire au pouvoir civil 1867.1963, document interne, Service historique, ministère de la Défense nationale, Ottawa, 1973.

31. «L'Acte concernant la police du Canada» (3l Vict. c.74) permet la nomination au Canada de commissaires habilités à nommer des constables. Cette loi donne naissance la même année à la seule police du gouvernement central, une simple garde des édifices gouvernementaux appellée la «Police du Canada» ou «Police du Dominion».

32. Cette force sera finalement organiscéc en 1873. À ce sujet, voir S.W. Horrail, «Sir John A. Macdonald and the Mounted Police Force for the Northwest Territories». Canadian Historical Review, vol. LIII, $\mathrm{n}^{\circ} 2$, juin 1972, p.179-200.

33. Le Manitoba crée une police provinciale en 1870 , et la Colombie en 1871 , peu de temps après avoir joint la Confédération.(Notons que dans cette dernière province, une police gouvernementale existait depuis 1858). À Terre-Neuve, colonie britannique, les autorités mettent sur pied la Newfoundland Constabulary à Saint-Jean, en 1871, après le départ des militaires. À ce sujet, on peut consulter la brève synthèse de William et Nora Kelly, dans Policing in Canada, Macmillan of Canada, 1976, p.1-27; C.K. Talbot, C.H.S. Jayewardene, et T.J. Juliani, op.cit.

34. Legislature d'Ontario. Bill $n^{\circ} 142,23$ janvier 1869. Bruno Deshaies, Évolution des Etats du Québec et de l'Ontario entre 1867 et 1871, thèse de doctorat, Histoire, Université de Montréal, 1973, p.151-152.

35. Selon le premier ministre Chauveau, DALQ 1869-70. 
conflits ouvriers, seulement quatre municipalités requièrent ses services en permanence, sans compter Québec, et deux autres temporairement, entre 1870 et 1878. Les premières sont Lévis, Hull, Sherbrooke, Oka, et les secondes, Buckingham et Sillery. Notons que ces municipalités, à l'exception d'Oka, sont parmi les plus populeuses de la province et des centres régionaux importants du réseau de transport ferroviaire et de l'industrie du bois. Mais dans un contexte de crise économique et de restrictions financières, l'avenir du système semble incertain. En 1877 , un coup fatal lui est porté lorsque, mettant fin à des négociations difficiles, la ville de Québec refuse d'augmenter sa participation au financement ${ }^{36}$. Le gouvernement riposte en retirant la police provinciale du territoire municipal. Le Bureau de police de Québec reprend ses activités et la police municipale est rétablie. La police provinciale fonctionne encore une année avec un effectif réduit, jusqu'au moment où, en 1878, le nouveau gouvernement libéral décide de l'abolir, respectant sa promesse électorale de couper dans les dépenses publiques. Bien que la loi constituante de la police provinciale ne soit pas abrogée, l'expérience du système de police s'achève donc abruptement, après huit ans d'un succès mitigé.

\section{8-1899: UNE MODESTE POLICE GOUVERNEMENTALE}

Le retour des conservateurs en 1879 n'entraîne pas la résurrection de la police provinciale. Le projet ambitieux de 1870 semble bel et bien abandonné. On lui préfère une modeste alternative qui permettrait au gouvernement d'assurer la surveillance de ses édifices et l'assistance aux tribunaux et aux organismes provinciaux, comme le faisait à Ottawa la «police du Dominion» fédérale. On retient les services de policiers qui sont pendant quelque temps intégrés au corps municipal et placés sous l'autorité du Bureau de police, conformément à une entente conclue par les libéraux en 1878. Le gouvernement conservateur se ravise en 1883, jugeant que ses intérêts étaient mal servis et reprend à compter de 1884 le contrôle de ses constables. Ceux-ci, une dizaine, sont placés sous le contrôle d'un nouveau commissaire de police nommé en vertu de la loi de 1870, Alexandre Chauveau, juge des sessions et membre du Bureau de police de Québec depuis 1880.

Au cours des années suivantes, cette police provinciale aux dimensions réduites s'éloigne du modèle de gendarmerie favorisé quelques années plus tôt. En raison des tâches qui lui sont confiées - la garde des nouveaux édifices parlementaires qui relèvent du département des 
Travaux publics, mais surtout la signification d'avis gouvernementaux, de pièces de procédure, et les enquêtes criminelles en province - la police provinciale devient de plus en plus le bras exécutif du Département du procureur général. Par conséquent, en 1896 et en 1897 , l'autorité sur la police provinciale, de même que la responsabilité des questions policières, sont retirées au Secrétariat provincial, pour être confiées au département du Procureur général ${ }^{37}$. Cette évolution suit une pratique observée dans l'administration publique au tournant du siècle, alors que l'application de certaines lois et de certains règlements, surtout lorsqu'ils sont liés à la perception de revenus, tend à être confiée non pas à un seul organisme de contrôle mais aux ministères concernés, et à des groupes de fonctionnaires dotés de pouvoirs d'enquête et de saisie. Ces groupes d'agents sont souvent désignés par le terme «police» bien que leurs pouvoirs soient plus restreints. Ainsi apparaît à Montréal au début des années 1880 une «police du revenu provincial» sous l'autorité du département du Trésor, chargée de la perception des taxes indirectes en vertu de la loi des «licences». La police provinciale devient en somme la police du département du Procureur général, comme celles du revenu et de la circulation seront au début du $20^{e}$ siècle celles du Trésor et de la Voirie ${ }^{38}$. Une loi de 1899 adoptée par le nouveau gouvernement libéral vient confirmer cette décision ${ }^{39}$. Elle abolit le poste de commissaire de police, rompant avec la tradition qui donnait à un magistrat la supervision des polices gouvemementales. Le procureur général, et surtout l'assistant procureur général, seront désormais, et pour longtemps, les premiers concernés par la gestion de la police provinciale ${ }^{40}$.

\section{9-1922: UNE POLICE PROVINCIALE ET DES POLICES GOUVERNEMENTALES}

Avant la Première Guerre mondiale, la Police provinciale a, en dépit de son nom, une aire d'action relativement réduite. Elle intervicnt surtout dans les environs de la capitale et dans l'est de la province, à partir de son quartier-général situé au sous-sol du Parlement. Bien que

37. Arrêté en conseil $n^{\circ} 302,23$ juillet 1896 ; 60 Vict. chap. 22.

38. Au début des années 1910, des «officiers de vitesse» sont nommés pour faire respecter la loi de 1906 sur l'immatriculation des voitures et l'obtention de permis de conduire, et recueillir les amendes; le service est transféré au département de la Voirie en 1924. Notons qu'en 1920-21, les revenus d'immatriculation et de permis représentent $9 \%$ des revenus provinciaux. J.I. Gow, op.cit, Appendice 2, p.394.

39. Loi concernant la police provinciale, 1899, 62 Vict. c.31.

40. Notons que, de 1905 à 1959, tous les premiers ministres sauf Adélard Godbout occupent le poste de procureur général. 
son effectif croisse au cours de ces années, son évolution est lente. Cela s'explique par la décision de confier l'application de certaines lois à des agences de contrôle autonomes et non à une direction unique. Certains phénomènes marquants dans l'histoire du contrôle policier nordaméricain, comme l'apparition de l'automobile et le resserrement des contrôles sur le commerce de l'alcool, ont donc très peu d'impact sur la petite Police provinciale de Québec. Les polices de la «circulation» et «des liqueurs» s'en chargent ${ }^{41}$. De même, les problèmes plus aigus de la région de Montréal en matière de criminalité amènent la formation entre 1905 et 1908 d'un «Bureau des détectives provinciaux» dans cette ville où le gouvernement engage depuis quelques années un détective privé ${ }^{42}$. Ce service relève des tribunaux et du département du Procureur général, comme la Police provinciale de Québec, mais n'entretient pas avec cette dernière de liens administratifs directs.

L'après-guerre et la prospérité modifient rapidement les conditions qui avaient déterminé la lente progression du système de police québécois depuis plusieurs décennies. Divers facteurs locaux, comme une vague de meurtres, les mesures de prohibition, les campagnes d'épuration des polices municipales ${ }^{43}$, se conjuguent aux inquiétudes provoquées par la révolution bolchévique, la grève générale de Winnipeg en 1919, pour stimuler des changements importants dans le système de police canadien. En 1919, le gouvernement fédéral fusionne la Dominion Police et la NWMP en une seule police nationale, la Gendarmerie royale du Canada, qu'il se propose de mettre à la disposition des provinces. En 1921, le gouvernement de l'Ontario donne une reconnaissance légale à l'organisation de sa police provinciale dont l'existence remontait à 190944 .

41. La «police des liqueurs» est créée en 1921 sous le nom de «service de surveillance» de la Commission des liqueurs, laquelle est chargée au même moment du contrôle de la vente d'alcool.

42. Le gouvemement confiait des enquêtes criminelles à la Canadian Secret Service et son directeur Silas H. Carpenter depuis la fin des années 1880. En 1900, un de ses détectives, Kenneth Peter McCaskill, est nommé «détective en chef du gouvernement».

43. À Montréal, une campagne amorcée en 1918 contre la corruption de la police conduit à la création d'une commission d'enquête connue sous le nom de Commission Cannon. Une enquête semblable s'était tenue en 1909. Voir au sujet des commissions d'enquête, J.-P.Brodeur, La délinquance de l'ordre, Cahiers du Québec, collection Droit et Criminologie, Hurtubise HMH, Montréal 1984.

44. En Ontario, la fonction de policier provincial existait depuis 1877 sans être attachée à une administration policière centralisée, mais plutôt aux autorités de comté. Un véritable corps de police, avec «superintendents» et quartier-général à Toronto, est organisé en 1909. D.D. Higley, op.cit. 
Le gouvernement québécois, qui est opposé à l'intrusion fédérale dans la juridiction provinciale sur la police, ne tarde pas à suivre, au moment où l'enlisement de l'enquête sur le meurtre d'une jeune fille de Québec, Blanche Garneau, suscite de fortes critiques dans le public. Cette affaire mobilise si bien l'opposition que le gouvernement libéral met sur pied à l'automne 1922 une Commission royale d'enquête sur l'administration de la justice afin de vider l'affaire 45 . Dans les mois qui précèdent, précisément en mars 1922, il réorganise sa police en créant un organisme connu sous les noms de «Police provinciale» ou «Sûreté provinciale» qui absorbe le Bureau des détectives provinciaux de Montréal et la Police provinciale de Québec ${ }^{46}$. Des détectives et des constables sont installés dans les deux divisions de la Sûreté, l'une ayant son quartier-général à Québec, et l'autre à Montréal. Chacune est dirigée par un chef sous l'autorité directe de l'assistant procureur général et du procureur général. Les salaires des policiers connaissent à cette occasion des augmentations sensibles, à Montréal d'abord. La loi démontre l'importance que prend alors la région de Montréal dans le contrôle policier gouvernemental, ce qui se traduira dans les années suivantes en ressources et en progrès matériels plus rapides que dans la division de Québec.

\section{2-1936: VERS UNE POLICE GOUVERNEMENTALE UNIFIÉE}

Les modifications apportées par la loi de 1922 n'éliminent pas complètement certaines caractéristiques de l'ancien sytème: les divisions fonctionnent pratiquement comme des corps séparés, la formation et l'entraînement des policiers et détectives se fait sur le terrain, l'embauche donne parfois plus d'importance aux affiliations politiques qu'à la compétence. Pourtant, à la fin de la décennie, le département semble touché par le mouvement nord-américain qui fait la promotion d'une administration policière inspirée du modèle militaire ${ }^{47}$. Du moins peut-on le penser lorsque, suite à la démission du chef de la division de

45. Les adversaires du gouvernement libéral dénoncaient l'incompétence des détectives gouvernementaux, donnant pour preuve une longue série de meurtres impunis. Ils accusaient le pouvoir politique d'avoir entravé la justice pour protéger les suspects, que la rumeur disait être fils de députés. La commission rejettera ces accusations. Voir Commission royale in re Administration de la Justice en rapport avec I affaire Blanche Garneau, ANQQ, Justice, art. 434, dossier 6796 ; Commissions d'enquête, art. 24.

46. Loi amendant les statuts refondus, 1909, relativement a la Police provinciale, 12 Geo.V, c. 69.

47. R.M. Fogelson, op. cit. 
Montréal, Dieudonné-Daniel Lorrain ${ }^{48}$, le gouvernement nomme pour le remplacer un avocat libéral, Maurice-Charles Lalonde, qui a servi dans l'armée, et dont les idées sur la police rejoignent celles de ses homologues américains. En effet, le mémoire qu'il soumet à la suite d'un voyage d'étude en Europe et dans l'Ouest canadien trace le plan de réformes qui seront accomplies au cours des trente années suivantes. La police provinciale doit être selon lui non seulement une force répressive mais aussi une force préventive agissant dans les régions dépourvues de service de police. Le processus de sélection des policiers doit être révisé, la discipline renforcée et encadrée par un code et un conseil de discipline, et la formation assurée par une École de police. Enfin, un commissaire de police prendrait la direction des deux divisions, et l'effectif serait augmenté.

Au moment où le régime libéral doit faire face à une opposition conservatrice plus agressive et à une crise économique majeure, plusieurs de ces propositions sont retenues par le département du Procureur général. Le poste de «chef de la Sûreté provinciale» est créé à Montréal et confié à Lalonde; les règlements concernant la police provinciale sont refondus, son personnel reclassifiét9. On assiste à une croissance considérable de son effectif, de même qu'à l'augmentation des salaires ${ }^{50}$. Enfin, on prépare la fusion des polices gouvernementales en permettant qu'elles soient transférées sous le contrôle du procureur général et de la Sûreté provinciale51. Entre 1930 et 1936, divers progrès accompagnent ces multiples mesures: formation d'escouades de la moralité, améliorations à l'équipement, ouverture d'un poste en Abitibj $^{52}$, création d'un service d'enquêtes criminelles à Québec. Notons que la division de Québec est placée à compter de 1931 sous la direction de l'officier de milice et comptable Léon Lambert, un partisan de la discipline. Il s'applique dès lors à réorganiser un corps de police

48. Ce détective de formation était chef depuis 1917. On le soupçonnait d'avoir utilisé les fonds publics à des fins personnelles.

49. Loi modifiant la loi de la police et du bon ordre, 1932, 22 Geo. V, c. 66.

50. Grosso modo, l'effectif à Montréal passe de 30 à 70 détectives et constables de 1929 à 1936, celui de Québec de 40 à 70.

51. Loi modifiant la Loi de la police et du bon ordre relativement à la prévention et à la répression d'infractions à des lois particulières, 1934, 24 Geo., c.50. Le service de surveillance de la Commisssion des liqueurs et la Police de la route passeront sous l'autorité du ministre et du chef de la Sûreté, en 1934 et en 1936 respectivement.

52. C'est dans cette région qu'était installé, depuis 1925 , le seul détachement de policiers provinciaux hors de Québec et de Montréal. Il devait voir principalement au contrôle du commerce de l'alcool et de la prostitution dans les zones d'exploitation minière où travaillaient de nombreux ouvriers immigrants. L'importance du détachement, ou «patrouille d'Abitibi» s'accrôt au début des années 30, au moment où la région connaît des manifestations soutenues par les communistes et des grèves importantes. 
qu'il juge à son arrivée «inefficace», «sans âme et sans tête», et voit à instaurer un entraînement pour les recrues et le personnel régulier, un précédent.

\section{6-1960: UNE POLICE VÉRITABLEMENT PROVINCIALE}

Ni la crise économique, ni l'élection de l'Union nationale en 1936 n'entravent ce mouvement. Le parti de Maurice Duplessis pousse encore plus loin les réformes entreprises sous le régime précédent. Le renvoi massif des policiers provinciaux de la division de Montréal en septembre 1936 inaugure une période de réorganisation intense. Elle s'accomplit d'abord sous la direction du chef Philippe Aubé, un avocat qui se charge de passer le personnel au crible, et à compter de l'automne 1937, sous le colonel P.A. Piuze, un ancien gouverneur du pénitencier de Saint-Vincent-de-Paul, et un état-major composé d'avocats et d'ex-militaires. Amorcée par la mise sur pied d'une école d'entraînement pour tous les policiers provinciaux, la réforme unioniste trouve sa pleine expression dans une loi sanctionnée en avril 193853. En effet, cette loi réorganise en profondeur la Sûreté provinciale du Québec et complète l'intégration des polices gouvernementales. Le corps de police est divisé en quatre «branches» dont les fonctions correspondent à celles des anciens services gouvernementaux: la police judiciaire, la gendarmerie, la police de la route, et la police des liqueurs, chacune portant un uniforme distinct. De plus, et c'est une première dans l'histoire de la Sûreté, des postes et sous-postes sont ouverts dans tous les comtés de la province. Jusqu'alors, à l'exception du poste de l'Abitibi, seuls les officiers des liqueurs et de la route étaient postés hors de Québec et de Montréal, du moins pendant la belle saison. En 1938, après l'intégration des polices de la route et des liqueurs, la Sûreté provinciale compte 565 policiers. Notons que c'est aussi en 1938, qu'est adoptée la «Loi du cadenas» ${ }^{54}$ qui lève les obstacles à la répression contre les communistes et conduit à la mise sur pied d'une escouade anti-subversive dans les deux divisions.

La nouvelle Sûreté provinciale subit quelques mois après sa réorganisation les contre-coups d'un revers politique inattendu. L'élection d'un gouvernement libéral en 1939 entraîne en effet de nombreux renvois dans la division de Montréal, surtout à la police de la route, ainsi que le retour de plusieurs policiers mis à pied en 1936. Piuze est muté et remplacé par un avocat, Marcel Gaboury, dont le titre de commissai-

53. Loi relative à la Sûreté provinciale, $2 \mathrm{Geo}$. VI, c.76.

54. Loi protégeant la province contre la propagande communiste, $1 \mathrm{Geo} . \mathrm{Vl}, \mathrm{c} .11$. 
re est changé pour celui de «directeur». L'entraînement est abandonné, on dissout l'escouade anti-communiste, le nombre de postes est réduit. Les divisions deviennent des «districts» et la police des liqueurs est détachée de la Sûreté provinciale pour être confiée à nouveau à l'autorité directe du département du Procureur général ${ }^{55}$. Le recul du modèle d'inspiration militaire devient plus évident en 1942 et 1943, lorsque des mesures sont adoptées pour intégrer les policiers provinciaux à la fonction publique, en les rendant admissibles au régime gouvernemental de retraite ${ }^{56}$, et en soumettant le processus d'embauche et de renvois aux contrôles de la nouvelle Commission du service civil ${ }^{57}$. De plus, en 1944, pour répliquer aux violentes attaques de l'Union nationale contre la Sûreté provinciale à Montréal sur la question du contrôle du jeu et de la prostitution, le gouvernement libéral institue une commission d'enquête sous la présidence du juge Lucien Cannon. Son rapport réfute les accusations portées contre la Sûreté provinciale, préférant s'arrêter sur les maladresses administratives du chef Aubé, nommé sous l'Union nationale, et vilipender les éditeurs d'un petit et virulent journal d'opposition à l'origine de la campagne contre la Sûretés8.

La création de la Commission du service civil semble salutaire puisque au retour au pouvoir de l'Union nationale en 1944, l'expérience des règlements de comptes post-électoraux de 1936 et 1939 ne se répète pas. La Sûreté amorce une période de croissance et de stabilité qui contraste avec les bouleversements des années précédentes. La prospérité d'après-guerre permet en effet d'accrô̂tre son efficacité et sa présence en améliorant son infrastructure (auto-patrouilles, système radio), en augmentant considérablement son effectif en particulier à la police de la route. En 1960, la Sûreté provinciale comptera 1234 employés, la police des liqueurs exclue ${ }^{59}$. On multiplie en outre le nombre d'escouades spécialisées et de postes de police.

Malgré quelques pas timides vers une meilleure coordination des branches, on est loin du commandement centralisé souhaité par la

55. Loi de la Sûreté provinciale et de la police des liqueurs, 1940, 4 Geo. VI, c. 56. Cette loi ne modifie pas les structures établies en 1938.

56. Depuis 1921, les policiers pouvaient en principe participer au régime de retraite, sous condition d'obtenir l'accord du ministre. La pratique d'offrir une demi-paie aux convalescents et retraités avait cependant prévalu, à la satisfaction des policiers qui ainsi ne voyaient pas leurs salaires grevés de contributions.

57. Les policiers sont dès lors tenus de s'abstenir de participer aux campagnes électorales, comme tout autre fonctionnaire provincial.

58. Ce journal est Le Moraliste, proche de l'entourage de Duplessis.

59. Soit à la police judiciaire et la gendarmerie, 319 employés à la division de Montréal, et 244 à la division de Québec : la police de la route compte 399 employés à Montréal et 272 à Québec. 
direction, sauf à la police de la route, car la coopération entre les branches demeure aléatoire ${ }^{60}$. Les districts sont pratiquement indépendants ${ }^{61}$, leur réputation contrastée. En effet, depuis l'arrivée du colonel Lambert à la division de Québec, celle-ci a la réputation d'être moins impliquée dans les manoeuvres électorales, et mieux administrée; son personnel a d'ailleurs échappé presque totalement aux purges des années 1936 et 193962 . La vacance du poste de directeur provincial entre 1950 et 1954, après la mort de son titulaire Me Joseph Paul Lamarche ${ }^{63}$, montre combien cette fonction n'avait plus l'importance que la loi de 1938 avait voulu lui accorder. Comme c'est le cas depuis le début du siècle, l'assistant procureur général assure la coordination administrative des deux divisions de la Sûreté, la gestion de chaque division étant l'affaire des directeurs adjoints. En 1954, le directeur adjoint à Montréal, Hilaire Beauregard, est le premier policier provincial à devenir directeur de la Sûreté sans que cela ne semble ajouter à son pouvoir et à son influence.

Au cours des années 1950, les adversaires du régime duplessiste attaquent plus vivement la police provinciale pour sa brutalité lors de certains conflits ouvriers (Asbestos, pour citer l'un des plus célèbres) et son travail partisan aux élections. À la même époque, on voit renaître au sein de la Sûreté un certain souci d'améliorer la formation des policiers. Depuis la guerre, les policiers qui avaient des compétences spéciales le devaient à leur expérience ou à leur propre initiative, ou à des cours donnés à l'École de la Gendarmerie royale et ailleurs. À partir de 1956-57, un entraînement des recrues et des policiers est repris. Le projet d'établir une véritable école prend forme après la mort de Maurice Duplessis, au moment où on songe à construire un nouveau quartier général à Montréal. Les cent jours du gouvernement de Paul Sauvé accélèrent ce timide renouveau et on voit le procureur général Antoine Rivard admettre à l'Assemblée nationale qu'il existe une section «politique» à la Sûreté, que cela doit cesser car la police doit être au-dessus

60. En 1945, les postes de directeur et assistant-directeur de la circulation à Québec et Montréal sont abolis et cette police placée sous l'autorité des directeurs adjoints de division. Notons que chaque branche porte un uniforme différent, sauf la police judiciaire qui est en civil, mais que les uniformes des policiers de la division de Québec se distinguent par certains détails de ceux de la division de Montréal.

61. Notons que chaque branche porte un uniforme différent, sauf la police judiciaire qui est en civil, et qu'en outre, les uniformes des policiers de la division de Québec se distinguent par certains détails de ceux de la division de Montréal.

62. Anecdote intéressante, en 1952, au cours d'un conflit de travail à Louiseville, le directeur adjoint Lambert tient à déclarer à la presse que ses policiers n'étaient pas présents au moment des incidents violents ayant impliqué la police provinciale.

63. Le policier de carrière Hilaire Beauregard hérite du poste en 1954. 
de tout soupçon d'ingérence politique, affirme-t-il. Des enquêtes internes sont entreprises ${ }^{64}$, et les promotions et les augmentations de salaires se multiplient. Mais voilà que l'élection de juin 1960 écarte les unionistes du pouvoir et donne la victoire au parti libéral qui entretient, au sujet de la Sûreté comme sur tous les aspects de l'administration publique, des projets plus ambitieux.

\section{0-1966: UNE RÉVOLUTION TRANQUILLE}

Les premières années de la Révolution tranquille à la Sûreté provinciale rappellent à plus d'un titre celles qui avaient suivi l'élection du premier gouvernement Duplessis en 1936. Outre les habituels renvois et démissions ${ }^{65}$, on assiste à un retour en force du modèle administratif militaire, qui semble répondre, plus que tout autre, au souci d'épuration et de rectification morale si cher aux réformateurs, tout en offrant des garanties éprouvées d'efficacité. La nomination d'un directeur issu de la Gendarmerie royale du Canada, Josaphat Brunet, est suivie d'un effort intensif pour évaluer le personnel en place et mettre l'embauche à l'abri de l'influence politique. On procède à une sélection plus rigoureuse du personnel (examens psychométriques, enquêtes, etc.), on crée une école de police pour les recrues. Plusieurs officiers à la retraite de la Gendarmerie royale sont associés à cette entreprise et accèdent au poste d'état-major, ce qui n'est pas sans soulever les critiques au sein de la police, qui se voit frustrée de promotions. En avril 1961, une loi donne à la Sûreté une structure plus centralisée, autour d'un état-major et d'un quartier général installés à Montréal et décloisonne les branches, qui deviennent de simples escouades. La loi crée en outre une École de police du Québect66. Un aspect essentiel, marquant, de cette loi est le renforcement de l'autorité du directeur général qui obtient des pouvoirs jusque-là réservés au ministre: ceux de recommander des nominations à tous les postes et de congédier sous-officiers et agents. Le contrôle du directeur sur la gestion de son personnel est d'autant plus grand que les policiers échappent désormais à l'autorité de la Commission du service civil, et que le pouvoir politique s'est engagé

64. Selon un ancien officier de la division de Montréal. L'information n'a pu être confirmée par des documents d'archives.

65. Ils touchent les policiers qui ont participé activement aux campagnes électorales, contrevenant à la loi du Service civil, ou qui ont commis d'autres irrégularités. La Sûreté mène elle-même des enquêtes internes. La police des liqueurs est particulièrement touchée. Un ancien directeur de cette police dira par la suite qu'elle servait à engager les «quêteux de job».

66. Statuts du Québec 1960-61, c. 18. 
publiquement à lui laisser sur ces questions les mains libres. Sous l'administration Brunet, pendant les premières années de la Révolution tranquille, la Sûreté provinciale fait du «rattrapage» plus qu'elle innove. Mise à part la dépolitisation du processus d'embauche, les progrès sont visibles du côté de son infrastructure (nouveau quartier-général, flotte automobile, système de télécommunications moderne, etc.) et de la formation.

\section{5-1974: UNE RÉVOLUTION TECHNOCRATIQUE}

Le directeur Brunet démissionne à la fin de 1965, dénonçant l'intervention du ministre de la Justice Claude Wagner qui avait refusé d'entériner une promotion et contesté son habitude de favoriser les retraités de la GRC. Au moment du départ de Brunet, la Sûreté s'est dégagée des attaches partisanes voyantes de l'ancienne police des régimes libéraux et unionistes pour présenter une image de neutralité politique, dans le sens partisan du terme, plus conforme à son statut de police d'État. L'impact négligeable de l'élection de l'Union nationale, en 1966, sur le personnel de la Sûreté confirme d'ailleurs que les moeurs politiques ont changé.

Le successeur de Brunet, Joseph-Adrien Robert, directeur de la police de Montréal sous l'adminitration Drapeau, s'est acquis une réputation de réformateur, et de «bureaucrate» ${ }^{67}$. Il arrive au moment où des tensions opposent les groupes d'officiers présents à l'état-major, anciens de la police provinciale et retraités de la GRC. C'est sous son administration, entre 1965 et 1968 , que la structure autoritaire mise en place en 1961 commence à chanceler. Les premiers craquements se font entendre lorsque les policiers provinciaux entreprennent de s'associer à l'automne 1965. Au moment où la filière politique ne peut plus comme autrefois transmettre et défendre leurs réclamations individuelles, l'accroissement du pouvoir de la direction et une discipline sévère poussent certains policiers à former en 1965 l'Association des policiers provinciaux du Québec. Après avoir obtcnu sa rcconnaissance officielle en $1966^{68}$, l'APPQ démontrera sa force à plusieurs reprises ${ }^{69}$, et deviendra un interlocuteur écouté de la direction et du ministère.

67. Le magazine Maclean, mars 1966, p.32. Le directeur Robert s'était fait connaître comme un réformateur du service de police de Hull (1937-1960) et de Montréal (1961-1965).

68. Une première tentative de s'associer avait échoué en 1961, les policiers hésitant à se commettre. La loi de 1961 avait répondu à certaines attentes en améliorant de façon notable leurs conditions salariales.

69. Par divers moyens de pression, la grève étant interdite. En 1971, pour le paiement d'heures supplémentaires faites lors de la crise d'octobre, en 1977 pour la patrouille à deux policiers, en 1984-85, pour les salaires. 
La seconde secousse est portée par le gouvernement et la direction elle-même. Lancée par l'administration Robert, elle atteindra toute sa force de 1969 à 1973, sous le directeur Maurice St-Pierre. Celui-ci, auparavant assistant directeur de la police de Montréal, cherche à atténuer le climat de méfiance qui affecte encore son état-major. Son administration participe à la volonté du gouvernement d'augmenter l'efficacité de la Sûreté face à la criminalité et sa présence sur le territoire québécois. C'est en prenant la voie technocratique et bureaucratique, comme l'administration publique québécoise, que la Sûreté connaît ce qui peut être considéré comme sa seconde révolution tranquille. Celleci se distingue du grand ménage autoritaire fait par l'administration Brunet, non par ses objectifs - efficacité des opérations et qualité du personnel - mais par les moyens d'y arriver: des méthodes scientifiques de gestion empruntées à l'entreprise privée.

En effet, à la fin de 1967 et au cours de l'année suivante, au moment où est préparé et présenté le projet de loi 14 quj voit à réglementer certains aspects du système policier québécois ${ }^{70}$, le ministère et la direction engagent une firme de conseillers en gestion, Raymond Ducharme et associés ${ }^{71}$, afin d'étudier les structures et le fonctionnement de la Sûreté du Québec ${ }^{72}$. Après de mutiples consultations avec les parties concemées, l'étude du fonctionnement de corps de police canadiens et américains, des visites dans les postes et escouades, et des centaines d'entrevues avec des agents et des officiers, la firme dépose plusieurs rapports sur les structures, le personnel et l'administration de la Sûreté. Elle constate les faiblesses de l'organisation mise en place en 1961: trop grande autonomie des deux divisions de Québec et Montréal, confusion créée par la multiplicité des niveaux hiérarchiques, le morcellement des fonctions et le caractère «nébuleux» des politiques et pratiques administratives, caractère arbitraire des promotions, déficiences dans le processus d'embauche et la formation, et enfin, statut

70. Cette loi refond les dispositions législatives ayant trait au maintien de la paix, de l'ordre et de la sécurité publique. Elle définit les champs de compétence des divers corps de police oeuvrant au Québec, et étend la juridiction du policier municipal à l'ensemble du territoire provincial. Elle prévoit la création d'une «Commission de police du Québec» destinée à «favoriser la prévention du crime et l'efficacité des services de police au Québec», celle d'un service central de renseignements policiers, et enfin, d'un Institut de police pour la formation de toutes les recrues policières.

71. La furme s'identifie ainsi : «Ingénieurs en organisation et méthode. Conseillers en gestion des entreprises». Si l'exercice est une première pour le corps de police provincial, il fait pratiquement partie des coutumes au sein de l'administration et du service de police de Montréal depuis le début du siècle. de 1968 .

72. Telle est la nouvelle désignation de la Sûreté provinciale selon la Loi de police 
inférieur des fonctionnaires. Pour y remédier, la firme élabore un projet d'envergure qui vise à rationaliser les politiques et pratiques selon le méthodes modernes de gestion. «À notre avis, écrit l'ingénieur Raymond R. Ducharme, ce projet va faire époque dans l'histoire de la Sûreté du Québec, à cause de l'orientation nouvelle que nous voulons donner aux activités policières et de la qualité des hommes qui seront éventuellement appelés à épauler cet effort» ${ }^{73}$.

La réorganisation qui s'amorce ne sera pas entravée par la défaite des unionistes en 1970. En effet, le gouvernement libéral de Robert Bourassa endossera le projet en l'intégrant dans un projet touchant l'ensemble de l'organisation policière québécoise ${ }^{74}$. De 1968 à 1973, la direction de la Sûreté du Québec met en oeuvre, après les avoir étudiées, les recommandations des spécialistes. En ce qui concerne l'administration et les politiques, on veut assurer une plus grande autonomie administrative de la direction générale face au ministère; en matière d'opérations, on cherche à accroître la capacité des entités régionales de réagir rapidement aux phénomènes criminels locaux. À cette fin, l'administration au Grand quartier général à Montréal est restructurée autour de cinq grandes directions sous des directeurs adjoints : planification, personnel, administration, services techniques, opérations. Des techniciens et professionnels civils sont embauchés, et des policiers formés aux méthodes de gestion. Les entités régionales traditionnelles divisions, sous-divisions et sections - sont redessinées de façon à épouser les limites des régions administratives gouvernementales. Au nombre de huit, chacun des nouveaux «districts» constitue un véritable commandement régional sous la direction d'un officier qui porte le titre de commandant; il a son propre quartier général et peut compter sur des services spécialisés jusque-là concentrés à la direction générale, ou au quartier de division. L'informatique doit être un outil essentiel de cette régionalisation puisqu'elle permet d'évaluer les résultats des opérations locales et d'avoir accès à une centrale de renseignements ${ }^{75}$.

L'implantation des districts commence à l'automne 1970 par l'établissement de deux districts-pilotes, (Bas-Saint-Laurent et Estrie); satisfaits de l'expérience, on inaugure en juillet et en août 1973 les six autres districts policiers. Cette délégation des pouvoirs, des services

73. ASQ, R.R. Ducharme à J.-J. Bertrand, ministre de la Justice, 17 juillet 1968.

74. Voir le livre blanc du ministre de la Justice de l'époque, Jérome Choquette, $\mathrm{La}$ police et la sécurité des citoyens, Éditeur officiel du Québec, [1970].

75. Le Centre de renseignements policiers du Québec (CRPQ) est mis sur pied par la Sûreté en collaboration avec la Commission de police, selon le mandat donné par la Loi de police de 1968. Il sera opérationnel à compter de juin 1974. 
administratifs, et des outils de gestion s'accompagne en contrepartie d'un resserrement des contrôles. L'autorité de la direction générale s'exerce par l'entremise d'une machine administrative anonyme, et un manuel de directives, produit de l'expérience policière et du savoir des spécialistes et gestionnaires civils. La conformité des pratiques dans les districts est assurée par des services de la direction générale (par exemple, celui de la «vérification»), par les commandants de districts, ainsi que par les «surveillants» et les «lecteurs». À la fín de cette période de restructuration, la Sûreté compte près de 5000 employés.

Maurice St-Pierre quitte la Sûreté en 1973 au moment où la restructuration est bien en place. Sous les administrations suivantes, celle de Paul A. Benô̂t (1973-1974), et celle de Jacques Beaudoin (1974 à 1988) $)^{76}$, tous deux issus des rangs de la police provinciale, la Sûreté ne dévie pas de l'orientation technocratique donnée en 1968. Cette période en est une de rodage et de perfectionnement de l'administration et des opérations policières 77 , mais non de bouleversements administratifs comme les années 1960, même si la récession des années 1980 entraîne des restrictions budgétaires et le gel de l'embauche de policiers (de 1981 à 1986) ${ }^{78}$.

À l'automne 1988, le directeur Beaudoin quitte son poste. C'est son collaborateur le plus immédiat, le directeur général adjoint à l'exécutif, Robert Lavigne, qui prend sa place. À la différence de ses prédécesseurs, il a fajt toute sa carrière dans la Sûreté depuis 1962, de patrouilleur à directeur général adjoint aux opérations et à la surveillance du territoire. Et c'est en grandes pompes que les générations de policiers embauchés après 1960 souligneront cet événement dans une salle du Stade olympique le 25 novembre 1988.

76. Il s'était surtout fait connaître à l'Institut de police du Québec, et au service du personnel (Formation et perfectionnement), ce dernier étant un des piliers de la nouvelle structure.

77. La conjoncture des années 1960-70 oriente les efforts vers les services de renseignements, les mesures d'urgence et les groupes tactiques d'intervention, la lutte au crime organisé, au commerce de la drogue, aux crimes économiques.

78. Un indice que la restructuration est acquise à la fin des années 1970 est le changement de la politique rédactionnelle de la Revue de la Sûreté du Québec, mensuel créé en 1971 pour diffuser et promouvoir les nouvelles politiques administratives. Délaissant le crédo technocratique, le revue se consacre à partir de 1979 aux phénomènes criminels et aux opérations policières ainsi qu'à la promotion d'une police présentée comme un service à la collectivité. Notons que la revue est rebaptisée Sûreté en 1982. 